\title{
Health relationships and disease relationships
}

If something like "health" existed in an individualized and singular manner, it could perhaps be described in the form of a transcendent and universal object that would contrast with a noun like "disease"; except that, because this does not exist, it also cannot be considered.

The historicity of health relationships and disease relationships that the population experience nonetheless rejects the possibility of reaching understandings, describing or interfering in what may come to be a state of healthiness or unhealthiness, outside of a perspective of togetherness, reciprocity and movement. In neither of these two cases can either dualist understandings or simple relativism, or even possibly obstinate culturalism, serve to explain why, in the light of the advances in production within the health sciences, the hospital and outpatient clinic structures of public nature continue to be inefficient. The efforts made by many professionals, technicians, intellectuals and managers, and by some politicians, are undeniable, but it also cannot be denied that the objectives that have attemptedly been pursued are far from being achieved. The volume and quality of attendance are not compatible with the academic knowledge that has been acquired locally, or with the proposal to take a comprehensive view of patients.

The expectation of something to be done that is incomplete and the perspective of insuperable difficulties have masked the possibilities that interactions with knowledge from different disciplines might allow. Divisions between academic disciplines only exist if we insist on not regarding them as artificial constructs: at the "wholesale" stage of the production of scientific knowledge, matters that are solely biological or psychological, or matters that are solely historical or statistical, become so because we need to fill out forms and because we need to write books and papers, among other reasons that establish professional divisions in modern societies.

This introduction has the purpose of presenting texts that here form a dossier united by topics within healthcare and education, bringing together different aspects of what is commonly understood to be public disclosure or dissemination of scientific knowledge. Each of the three papers described below brings out understandings regarding historical processes with the capacity to help in thinking and, with some effort, who knows, in building up healthcare practices through routes that are more inclusive.

The paper by André Mota, "Cleaning up the race through the hands of ruralist education: the case of the Butantan Rural School Group in the 1930s", discusses a set of educational proposals put forward during the time of the Vargas government, within its attempts to construct a strong state and, at the same time, a civilized nation. Hence, a link was made between childhood (the central point of educational actions) and stimulation of sanitary and moral concepts that would aid in molding populations originating in rural areas. In discussing the path taken by this project and its limits, Mota writes about a specific period and brings out arguments for a discussion that includes ethnographic dimensions, if we also wish to look at education through its bias of diffusion of technical knowledge, allied with the abstract nature of what is transmitted.

This is precisely the issue, of descriptive expression regarding the historical paths followed by Brazilian education and health, that is presented by the second study "Health education in $16 \mathrm{~mm}$ : audiovisual memory of the Special Public Health Service (SESP)", by Maria Cristina Soares Guimarães et al.

This paper provides news regarding the important work of recovering film collections and conserving them. It presents a set of films that were specially produced in the 1940s, with the aid of the Rockefeller Foundation, for disseminating healthcare knowledge, as a means of educating the public about various types of disease, particularly on tropical continents like Africa, Asia and Latin America.

Although educational movies remain little explored as source material for studies, they provide possibilities for achieving very diverse understandings. They assist both in comprehending the concepts of science, society and the public within a given period and place, and in furnishing clues regarding the 
formation of structures that define our notions of health and disease as delimited and individualized matters. This has a close relationship with the Western manner of imposing education, instituting knowledge and determining "worldviews".

The third and final paper in this set of studies is a text with the title "Collection of educational materials on Hansen's disease: a tool for memory and communicative practices", by Adriana KellySantos, Simone Souza Monteiro and Ana Paula Goulart Ribeiro.

Through describing and analyzing various types of support that had the aim of disseminating information on one specific disease, i.e. Hansen's disease, between 1975 and 2008, the paper brings out a debate that closes this small discussion cycle. The important conclusion indicated by this paper is that there is a persistent disconnection between educational practices and dissemination. The paper outlines the scarcity of follow-up regarding the "effectiveness of the strategies adopted", in light of the information policies to which different publics (patients, accompanying persons and vulnerable categories) are subject. At the same time, it provides indications of procedures and actions that have the capacity to diminish the distance between the public and the healthcare services when educational activities cease to be "vertical, unilateral and linear".

The questions raised by these papers lead us towards formulating certain understandings. In a general manner, a desire for sole and exclusive application of technical knowledge to the complexity of health and disease relationships, for example in relation to patients' social position and to the power of physicians or educators, may not be fully effective. Secondly, associations between scientists within the so-called human sciences and scientists responsible for production of biological knowledge may make it possible to discern understandings that generate associations that are more advantageous for everyone who is dedicated to this field, including patients. Finally, we could seek to study the network of relationships in such a way as to explain how, and with what costs and for which groups, it is of interest to maintain the discrepancy between the quality of production of scientific knowledge within healthcare and the insufficiency of the attendance provided by public services in Brazil.

Márcia Regina Barros da Silva Federal University of São Paulo 